\title{
ARTICLE
}

\section{Th22 cells are efficiently recruited in the gut by CCL28 as an alternative to CCL20 but do not compensate for the loss of Th17 cells in treated HIV-1-infected individuals}

\author{
Manon Nayrac ${ }^{1}$, Mary Requena ${ }^{2}$, Claire Loiseau ${ }^{1,9}$, Michelle Cazabat ${ }^{2}$, Bertrand Suc ${ }^{3,4}$, Nicolas Carrere ${ }^{3,4}$, Karl Barange $^{5}$, \\ Laurent Alric ${ }^{3,6,7}$, Guillaume Martin-Blondel ${ }^{1,3,8}$, Jacques Izopet ${ }^{1,2,3}$ and Pierre Delobel $\mathbb{I D}^{1,3,8}$
}

Gut $\mathrm{CD}^{+} \mathrm{T}$ cells are incompletely restored in most HIV-1-infected individuals on antiretroviral therapy, notably Th17 cells, a key subset in mucosal homeostasis. By contrast, gut Th22 cells are usually restored at normal frequencies. Th22 cells display a $\mathrm{CCR}^{+}{ }^{+} \mathrm{CCR} 10^{+}$phenotype and could thus respond to CCL20- and CCL28-mediated chemotaxis, while Th17 cells, which express CCR6 but not CCR10, depend on CCL20. Herein, we found that CCL28 is normally expressed by duodenal enterocytes of treated HIV1 -infected individuals, while CCL20 expression is blunted. Ex vivo, we showed that Th22 cells contribute to the reduction of CCL20 production by enterocytes through an IL-22- and IL-18-dependent mechanism. Th22 cells preferentially migrate via CCL20- rather than CCL28-mediated chemotaxis when both chemokines are available in the microenvironment. However, when the CCL20/CCL28 ratio drops, as in treated HIV-1-infected individuals, Th22 cells can migrate via the CCR10-CCL28 axis, as an alternative to CCR6-CCL20. This could explain the better reconstitution of gut Th22 compared with Th17 cells on antiretroviral therapy. Lastly, we assessed the relationships between the frequencies of gut Th17 and Th22 cells and inflammatory markers related to microbial translocation, and showed that Th22 cells do not compensate for the loss of Th17 cells in treated HIV-1-infected individuals.

Mucosal Immunology (2021) 14:219-228; https://doi.org/10.1038/s41385-020-0286-6

\section{INTRODUCTION}

HIV-1 infection causes a deep alteration of the intestinal mucosa. The gut contains numerous target cells for HIV-1, and thus supports a high level of virus replication throughout infection. ${ }^{1-3}$ It results in a severe depletion of intestinal $\mathrm{CD}^{+}{ }^{+} \mathrm{T}$ cells and in a loss of the mucosal barrier integrity, both of which are commonly considered as critical contributors to chronic inflammation and disease progression in HIV-1-infected individuals. ${ }^{4,5}$

Th17 cells are a key subset of $\mathrm{CD}^{+} \mathrm{T}$ cells in the intestinal mucosa where they play an important role in mucosal defense and homeostasis, but are also particularly targeted by HIV-1. ${ }^{6-11}$ Their depletion has been associated with microbial translocation from the gut lumen during HIV-1 infection. ${ }^{4,12-14}$ Th17 and Th22 cells share a pivotal role in the prevention of microbial translocation by stimulating epithelial cell renewal, maintaining the integrity of intestinal tight junctions, and producing antibacterial defensins. ${ }^{15-17}$ The functional differentiation of intestinal Th17 cells has been shown to be dependent on the interactions between the gut microbiota and mucosal cells, notably through IL-22 and serum amyloid A proteins. ${ }^{18-20}$

Th17 cells are characterized by the expression of the transcription factor RORyt, a $\mathrm{CCR} 4^{+} \mathrm{CXCR} 3^{-} \mathrm{CCR} 6^{+} \mathrm{CD} 161^{+}$phenotype, and a strong production of IL-17., ${ }^{6,21,22}$ Th22 cells share
CCR6 expression with Th17 cells but they differ regarding a higher expression of the transcription factor $A h R$, the additional expression of CCR10, and the preferential production of IL-22. ${ }^{15,23}$ Early in HIV-1 infection, both Th17 and Th22 cell subsets are massively depleted in the intestinal mucosa, and the associated loss of the intestinal immune barrier results in microbial translocation events. ${ }^{17,24-27}$

Despite prolonged effective antiretroviral therapy, the reconstitution of gut Th17 cells remains incomplete in most HIV-1infected individuals. ${ }^{26-28}$ In contrast to Th17 cells, the frequency of Th22 cells appears to be restored. ${ }^{25,26,29}$ We previously showed that the recruitment of Th17 cells in the duodenal mucosa is impaired in HIV-1-infected individuals receiving antiretroviral therapy due to an alteration of CCR6-mediated chemotaxis as the result of a reduced production of CCL20 by intestinal epithelial cells (IECs). ${ }^{28}$ We found that Treg cytokines (IL-10 and TGF- $\beta$ ) and Th1 cytokines (IFN- $\gamma$ and IL-18) reduce CCL20 production by enterocytes, while TNF- $\alpha$, IL-1 $\beta$, and IL-17A increase it. ${ }^{28,30}$

Th22 cells can migrate to the gut via CCR6- and/or CCR10mediated chemotaxis. ${ }^{15}$ CCL28, the main ligand for CCR10, is produced by IECs, but CCL28 expression in the intestinal mucosa of treated HIV-1-infected individuals is yet to be explored. ${ }^{31}$ The restoration of Th22 cells in the gut mucosa despite a reduced

\footnotetext{
${ }^{1}$ INSERM, UMR1043, Toulouse F-31300, France; ${ }^{2} \mathrm{CHU}$ de Toulouse, Laboratoire de Virologie, Toulouse F-31300, France; ${ }^{3}$ Université Toulouse III Paul Sabatier, Toulouse F-31300, France; ${ }^{4} \mathrm{CHU}$ de Toulouse, Service de Chirurgie Générale et Digestive, Toulouse F-31300, France; ${ }^{5} \mathrm{CHU}$ de Toulouse, Service d'Hépato-Gastro-Entérologie, Toulouse F-31300, France; ${ }^{6} \mathrm{CHU}$ de Toulouse, Service de Médecine Interne, Toulouse F-31300, France; ${ }^{7} \mathrm{RD}$ UMR152, Toulouse F-31400, France and ${ }^{8} \mathrm{CHU}$ de Toulouse, Service des Maladies Infectieuses et Tropicales, Toulouse F-31300, France

Correspondence: Pierre Delobel (delobel.p@chu-toulouse.fr)

${ }^{9}$ Present address: Centre for Molecular Therapeutics, Australian Institute of Tropical Health and Medicine, James Cook University, Cairns, QLD, Australia
}

Received: 21 September 2019 Revised: 19 February 2020 Accepted: 24 March 2020

Published online: 28 April 2020 
production of $\mathrm{CCL} 20$ by enterocytes could indicate that an effective CCR10-CCL28 axis represents a functional alternative for the recruitment of Th22 cells in treated HIV-1-infected individuals.

In this study, the frequencies of Th17 and Th22 cells, as well as the expression of CCL20 and CCL28, were measured in biopsies of the duodenal mucosa from HIV-1-infected individuals receiving prolonged effective antiretroviral therapy. Mechanisms underlying the regulation of CCL20 and CCL28 expression by enterocytes were next assessed by performing ex vivo cocultures between Th17/Th22 cells and IECs. Herein, we showed that CCL28 expression and Th22 cell frequency in the duodenal mucosa are similar between treated HIV-1-infected and uninfected individuals, contrasting with the reduced CCL20 expression and Th17 cell frequency observed in treated HIV-1-infected individuals. We demonstrated that IL-22 could interfere with CCL20 production by enterocytes via an IL-18-dependent mechanism and could thus contribute to blunt Th17 cells recruitment. Th22 cells could migrate via CCR6 or CCR10 depending on the CCL20/CCL28 ratio in the microenvironment, suggesting fine tuning of the recruitment of Th17 and Th22 cells in the gut mucosa. Lastly, we assessed the relationships between the frequencies of gut Th17 and Th22 cells and inflammatory markers related to microbial translocation, and showed that Th22 cells do not compensate for the loss of Th17 cells in treated HIV-1-infected individuals.

\section{RESULTS}

A preserved frequency of Th22 cells contrasts with the loss of Th17 cells in the duodenal mucosa of treated HIV-1-infected individuals We first assessed the frequencies of Th17 and Th22 cells in CD4 $T$ cells in the peripheral blood and the lamina propria of the duodenum of HIV-1-infected individuals on prolonged effective antiretroviral therapy. Th17 and Th22 cells were measured by flow cytometry on the basis of their surface phenotype (Fig. 1a). We found that the frequency of Th17 cells remained lower in the duodenal mucosa of treated HIV-1-infected individuals compared with uninfected controls, while it did not significantly differ in the peripheral blood $(2.8 \%$ vs. $2.3 \%, P=0.631$ in blood; $7.8 \%$ vs. $10.4 \%, P=0.0002$ in duodenum; Fig. $1 \mathrm{~b})$. The frequency of Th1Th17 cells was also significantly lower in the duodenal mucosa of treated HIV-1-infected individuals compared with uninfected controls (Supplementary Fig. S1).

By contrast, the frequencies of Th22 cells in $\mathrm{CD}^{+}{ }^{+} \mathrm{T}$ cells in the peripheral blood and duodenal mucosa were similar between treated HIV-1-infected individuals and uninfected controls $(0.5 \%$ vs. $0.7 \%, P=0.280$ in blood; $6.6 \%$ vs. $6.8 \%, P=0.971$ in duodenum; Fig. 1C).

The frequencies of Th17 cells in the peripheral blood and duodenal mucosa were not correlated $(\rho=-0.08, P=0.724$; Fig. 1d), while the frequencies of Th22 cells were positively correlated between both compartments $(\rho=0.51, P=0.020$; Fig. 1e). These results suggest significant differences between Th22 and Th17 cells regarding their recruitment, renewal, and/or survival rates in the duodenal mucosa of treated HIV-1-infected individuals.

Preserved expression of CCL28 contrasts with reduced expression of CCL20 by enterocytes in the duodenal mucosa of treated HIV-1infected individuals

The decreased frequency of Th17 cells in the duodenal mucosa of treated HIV-1-infected individuals could be in part related to their impaired recruitment through the CCR6-CCL20 chemotactic axis. $^{28}$ Th22 cells share CCR6 expression with Th17 cells but differ regarding CCR10 expression. We thus investigated whether CCL28, the main ligand of CCR10 produced by enterocytes, could efficiently drive Th22 cell recruitment as an alternative to the CCR6-CCL20 axis. CCL28 production was preserved in the duodenal epithelium of treated HIV-1-infected individuals compared with uninfected controls, as quantified by immunohistochemistry (quantification of CCL28 per surface area, $P=0.325$; Fig. 2). This contrasts with the reduced production of CCL2O by enterocytes in treated HIV-1-infected individuals. ${ }^{28}$

These results suggest that Th22 cell recruitment in the duodenal mucosa could depend on the CCR10-CCL28 axis while Th17 cell recruitment is blunted because of a defective CCR6-CCL20 axis.

Ex vivo, Th22 cells decrease CCL20 but not CCL28 expression by intestinal epithelial cells

To further explore the regulation of CCL20 and CCL28 expression by enterocytes, we used an ex vivo model of primary human IECs cultured on transwell inserts to assess the production of CCL20 and CCL28 by IECs.

CCL20 mRNA expression by IECs was increased up to sixfold after stimulation by IL-17A, the main Th17 cytokine, while it was decreased by twofold when stimulated with IL-22, the main Th22 cytokine ( $P<0.05$ for IL-17A and IL-22 stimulations; Fig. 3a). These data demonstrate that IL-17A and IL-22 have opposing effects on CCL20 expression by IECs. IL-17F had no significant impact on CCL20 expression (data not shown), whereas TNF- $\alpha$ and IL-1 $\beta$ strongly induced it, as already described. ${ }^{30}$

CCL28 mRNA expression by IECs was only weakly increased by IL-17A and IL-22 stimulation $(P<0.05$ for IL-17A and $P<0.01$ for IL22 stimulation; Fig. 3b), and not significantly modulated by IL-17F, TNF- $\alpha$, and IL-1 $\beta$ in our model of culture of primary human small intestine cells (data not shown).

We next assessed the global effect of the cytokines produced by Th17 and Th22 cells by coculturing IECs and sorted Th17 or Th22 cells, added in the bottom chamber of transwells to mimic interactions between IECs and lymphocytes in the intestinal mucosa.

Because of some overlap in the cytokines that can be produced by Th17 and Th22 cells, we first quantified the levels of IL-17A and IL-22 produced by each cell subset sorted on the basis of their surface phenotype. Th22 cells produced fourfold more IL-22 than Th17 cells did, while the inverse was found for IL-17A which was produced fourfold more by Th17 than by Th22 cells $(P=0.025$ for both comparisons; Supplementary Fig. S2a,b). In agreement with cytokine production, the expression of the $A h R / R O R c$ ratio was higher in Th22 than in Th17 cells $(P=0.009$; Supplementary Fig. S2c).

In coculture experiments, CCL20 mRNA expression was reduced by twofold in Th22:IEC cocultures relatively to Th17:IEC cocultures, while CCL28 mRNA expression was no different $(P=0.022$ for CCL20 and $P=0.575$ for CCL28; Fig. 3C), in agreement with the results of cytokines stimulation. These results were confirmed at a protein level, with a twofold reduction of the median production of CCL20 in Th22:IEC cocultures compared with Th17:IEC cocultures ( $P=0.038$; Fig. $3 \mathrm{~d})$, while CCL28 production was similar $(P=0.497$; Fig. 3e).

IL-22 and IL-18 cooperate in blunting CCL20 expression by IECs We previously demonstrated that IL-18 downregulates CCL20 expression by IECs. ${ }^{30}$ As already reported, IL-22 can induce IL-18 expression in IECs, suggesting that IL-22 may induce indirect downregulation of CCL20 via IL-18. ${ }^{32}$ Indeed, in our model of culture of primary human small intestine cells, IL-22 induced a tenfold increase of pro- $/ L-18$ mRNA expression by IECs $(P<0.05$; Fig. 4a). We thus assessed the respective effects of IL-22 and IL-18 in blunting CCL20 production by IECs in Th22:IEC cocultures. Blockade of IL-22 biological effect using IL-22 binding protein (IL22BP) almost totally abolished the downregulation of CCL20 expression in Th22:IEC cocultures relative to Th17:IEC (Fig. 4b). This demonstrates that IL-22 is required for CCL20 downregulation in Th22:IEC cocultures. However, the effect of IL-22 appears to be mostly indirect through the induction of IL-18 by IECs, as blockade 
a

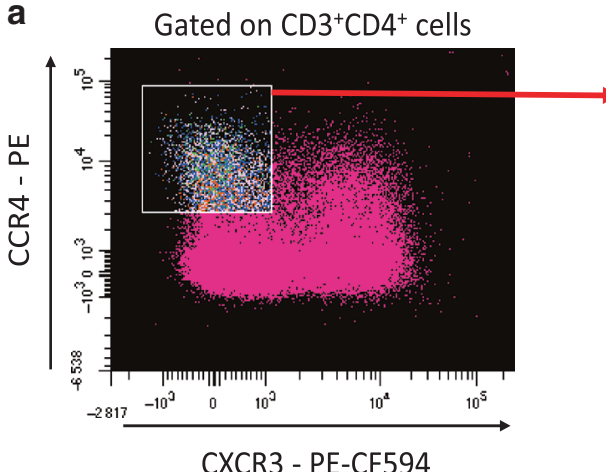

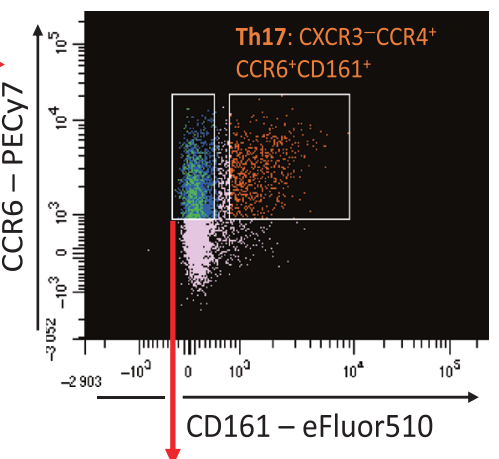

b
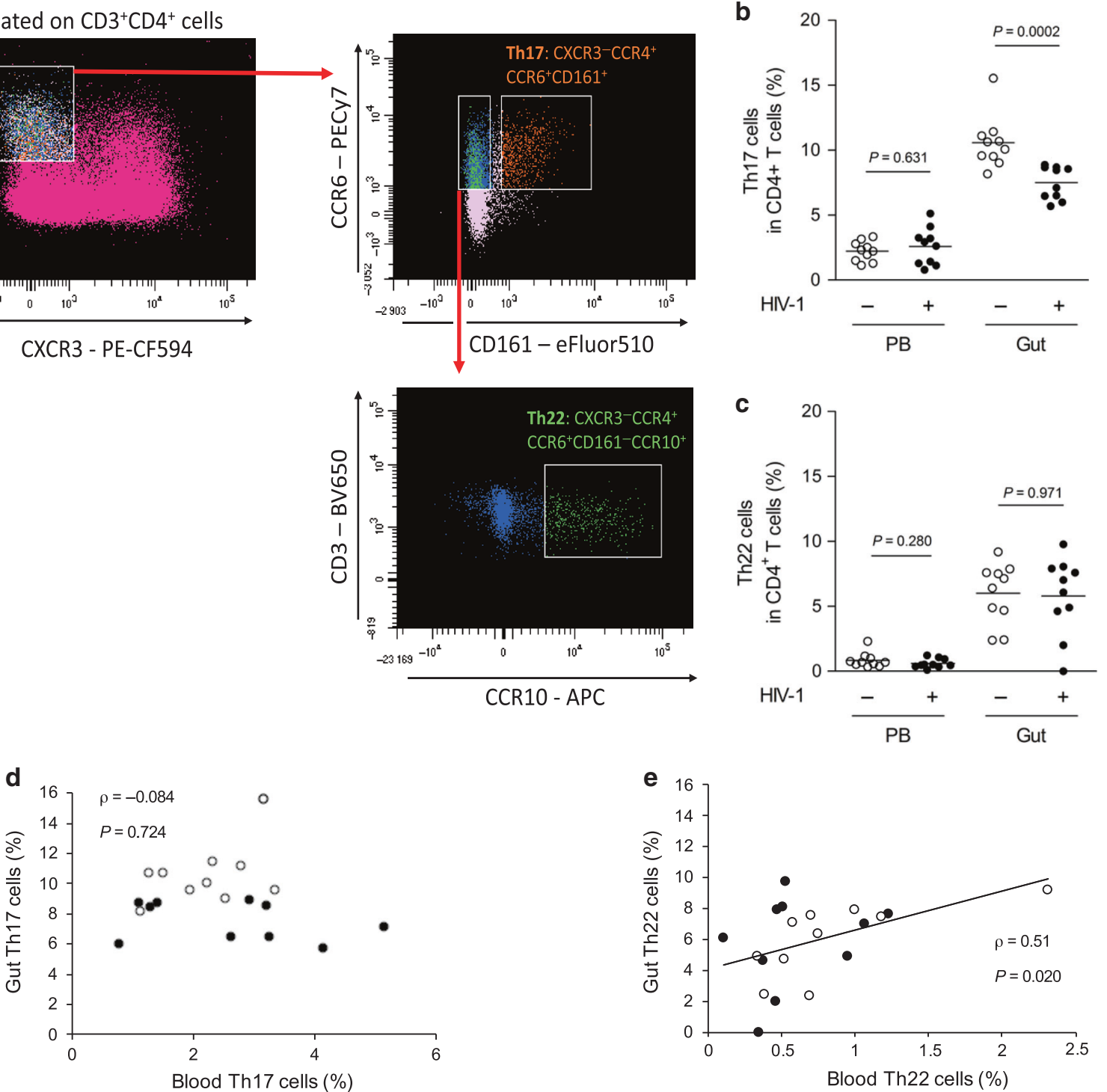

Fig. 1 Frequencies of Th17 and Th22 cells in CD4 ${ }^{+} \mathbf{T}$ cells of the peripheral blood and duodenal mucosa. a Flow cytometry gating strategy

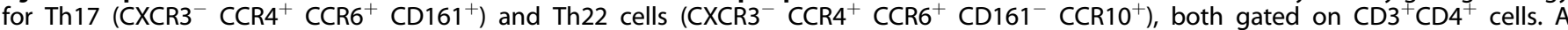
representative HIV-1-infected individual is shown. There were no significant differences in FACS gating between HIV-1-infected and uninfected individuals (data not shown). b Th17 and c Th22 cells frequencies in CD4 ${ }^{+}$T cells in the peripheral blood (PB) and duodenal mucosa of treated HIV-1-infected $(n=10)$ and uninfected individuals $(n=10)$. Percentage of cells was determined by flow cytometry. Groups were compared with the Wilcoxon's rank-sum test. Median bars are shown. Correlation between the peripheral blood and duodenal mucosa frequencies of $\mathbf{d}$ Th17 and $\mathbf{e}$ Th22 cells (ten treated HIV-1-infected and ten uninfected individuals). Each symbol represents one individual. $\mathrm{HIV}-1^{-}$, white symbol; $\mathrm{HIV}-1^{+}$, black symbol. Correlations were estimated by calculating Spearman's rank correlation coefficients.

of IL-18 biological effect using IL-18BP also abolished the downregulation of CCL20 expression in Th22:IEC cocultures relatively to Th17:IEC cocultures (Fig. 4b). Thus, IL-22 downregulates CCL20 expression in enterocytes via an IL-18-mediated, indirect mechanism.

Th22 cells migrate via CCR6 or CCR10 depending on the CCL20/ CCL28 ratio

Finally, we assessed the global effect of the chemokines present in the microenvironment of the intestinal mucosa on the recruitment of Th17 and Th22 cells. We performed cell migration assays to assess the chemotaxis of Th17 and Th22 cells using conditioned medium from Th17:IEC or Th22:IEC cocultures, reflecting a Th17 or Th22 microenvironment, respectively.

Th17 cell recruitment was significantly lower in a Th22 microenvironment than in a Th17 one $(P=0.025$, Fig. 5a), in agreement with our results showing that IL-22 reduced the production of CCL20 by enterocytes. By contrast, Th22 cell recruitment was similar in a Th22 microenvironment compared with a Th17 one ( $P=0.575$, Fig. $5 b)$. The median CCL20/CCL28 ratio was twofold higher in the medium of Th17:IEC vs. Th22:IEC cocultures ( 0.29 and 0.15 , respectively, $P=0.043$, Fig. $5 c)$.

Th17 cells recruitment depends on the CCR6-CCL20 axis as blocking the biological effect of the CCL20 chemokine by a neutralizing antibody significantly blunted Th17 cells recruitment $(P<0.05$ for all CCL20 blocking conditions), while blocking CCL28 did not have any effect, in either a Th17 (Fig. 5d) or Th22 microenvironment (Fig. 5e). CCR6 expression was downregulated in response to its occupancy by CCL20, while it was not modified by CCL28 (Supplementary Fig. S3), in agreement with the absence of interference of CCL28 in Th17 cells recruitment through the CCR6-CCL20 axis. 
Th22 recruitment appears to depend on the CCL20/CCL28 ratio in the microenvironment. When the $\mathrm{CCL} 20 / \mathrm{CCL} 28$ ratio was sufficient, i.e., in a Th17 microenvironment, Th22 cells migrated mainly via the

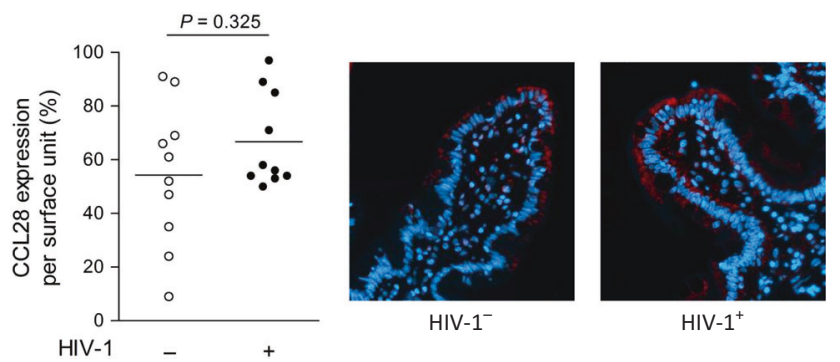

Fig. 2 Expression of CCL28 by enterocytes in the duodenal mucosa. CCL28 production in the duodenal mucosa of treated HIV1 -infected $(n=10)$ and uninfected individuals $(n=10)$. Chemokine expression was quantified per surface unit of epithelium using NISelement (Nikon). CCL28 (red) was stained by immunohistochemistry on an ApoTome (Zeiss, original magnification $\times 63$ ). Cell nuclei were counterstained (DAPI, blue). Representative treated HIV-1-infected and uninfected individuals are shown. Groups were compared with the Wilcoxon's rank-sum test. Median bars are shown.
CCR6-CCL20 axis, as blocking CCL20 significantly blunted their recruitment $(P<0.05$ for all $C C L 20$ blocking conditions), while blocking CCL28, alone or in addition to blocking CCL20, did not have any significant impact (Fig. 5f). By contrast, when the CCL20/ CCL28 ratio was too low, i.e., in a Th22 microenvironment, Th22 cells migrated mainly via the CCR10-CCL28 axis, as blocking CCL28 significantly blunted their recruitment $(P<0.05$ for all CCL28 blocking conditions), while blocking CCL20, alone or in addition to blocking CCL28, did not have any significant impact (Fig. $5 \mathrm{~g}$ ).

When varying the CCL20/CCL28 ratio, the migration rate of Th17 cells linearly increased in parallel with CCL20 concentrations, but was null in the presence of CCL28 alone, in agreement with the dependence of Th17 cells on CCL20- but not CCL28-mediated chemotaxis ( $P<0.0001$; Fig. 5 h). By contrast, Th22 cells migration could depend on both CCL20 and CCL28. When migration assay was performed with constant concentration of $\mathrm{CCL}_{2} 8$ and relatively low concentration of $\mathrm{CCL} 20$, the migration rate of Th22 cells remained constant (Fig. 5i). However, with higher concentrations of CCL20, the migration rate of Th22 cells progressively increased, suggesting that a significant threshold of CCL20 concentration is required for recruiting Th22 cells through the CCR6-CCL20 axis, in addition or in replacement of CCL28-mediated chemotaxis $(P=0.015$; Fig. 5i).

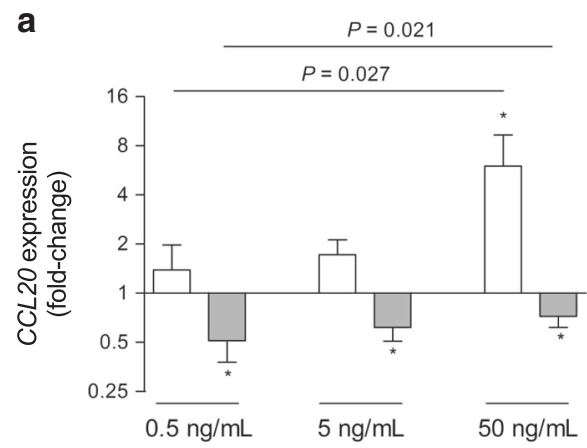

b

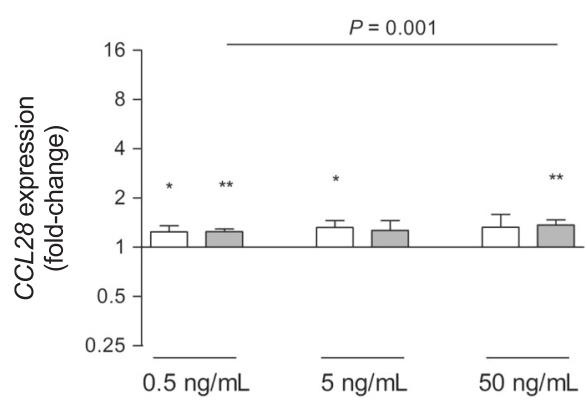

IL-17A $\square$ IL-22

C

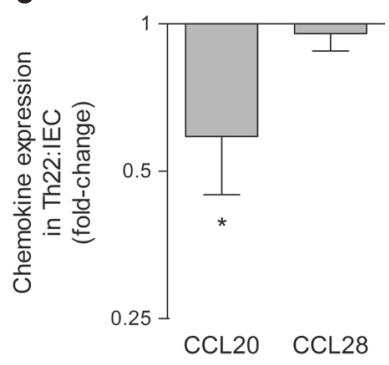

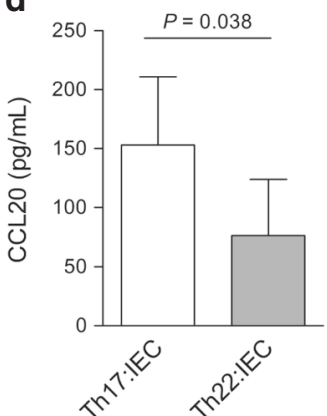

e

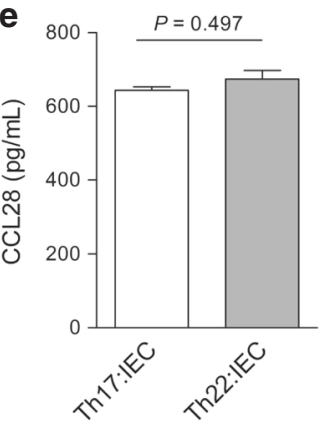

Fig. 3 Impact of the interactions between IL-17A/Th17 cells and IL-22/Th22 cells on CCL20 and CCL28 expression by enterocytes. Effect of IL-17A (white bars) and IL-22 (gray bars) on a CCL20 and b CCL28 mRNA expression by enterocytes. Monolayers of differentiated human primary enterocytes on transwell inserts were stimulated by $0.5,5$, and $50 \mathrm{ng} / \mathrm{mL}$ of cytokine. CCL20 and CCL28 mRNA was quantified in the enterocytes by qRT-PCR. CCL20 and CCL28 expression following cytokine stimulation was normalized relatively to the expression in unstimulated epithelial cells (set to 1) and expressed as fold change (log2 scale). Presented data were obtained from at least eight independent experiments performed with different donors. Cuzick's test for trend was used to compare chemokine expression across the increasing concentrations of cytokines (the corresponding $P$ value is shown on the line above the IL17-A and IL-22 bars); paired Wilcoxon's test was used to compare chemokine expression upon stimulation vs. unstimulated condition (intradonor pairing); $P$ value is shown above each bar; ${ }^{*} P<0.05 ;{ }^{*} P<0.01$. Means and SEM are shown. c Effect of Th22:IEC coculture on CCL20 and CCL28 mRNA expression by enterocytes. Enterocytes were cocultured with FACS-sorted Th22 or Th17 cells, added in the bottom chamber for $15 \mathrm{~h}$. CCL20 and CCL28 mRNA were quantified in the enterocytes by qRT-PCR. CCL20 and CCL28 expression in Th22:IEC coculture was normalized relatively to the expression in Th17:IEC coculture (set to 1) and expressed as fold change (log2 scale). Enterocytes production of d CCL20 and e CCL28 proteins in Th22:IEC and Th17:IEC cocultures. CCL20 and CCL28 were quantified in the bottom chamber by ELISA. Presented data were obtained from ten independent experiments performed with different donors. Paired Wilcoxon's test was used to compare CCL20 and CCL28 in Th22:IEC vs. Th17:IEC cocultures (intradonor pairing); ${ }^{*}, P<0.05$. IEC, intestine epithelial cells. Means and SEM are shown. 

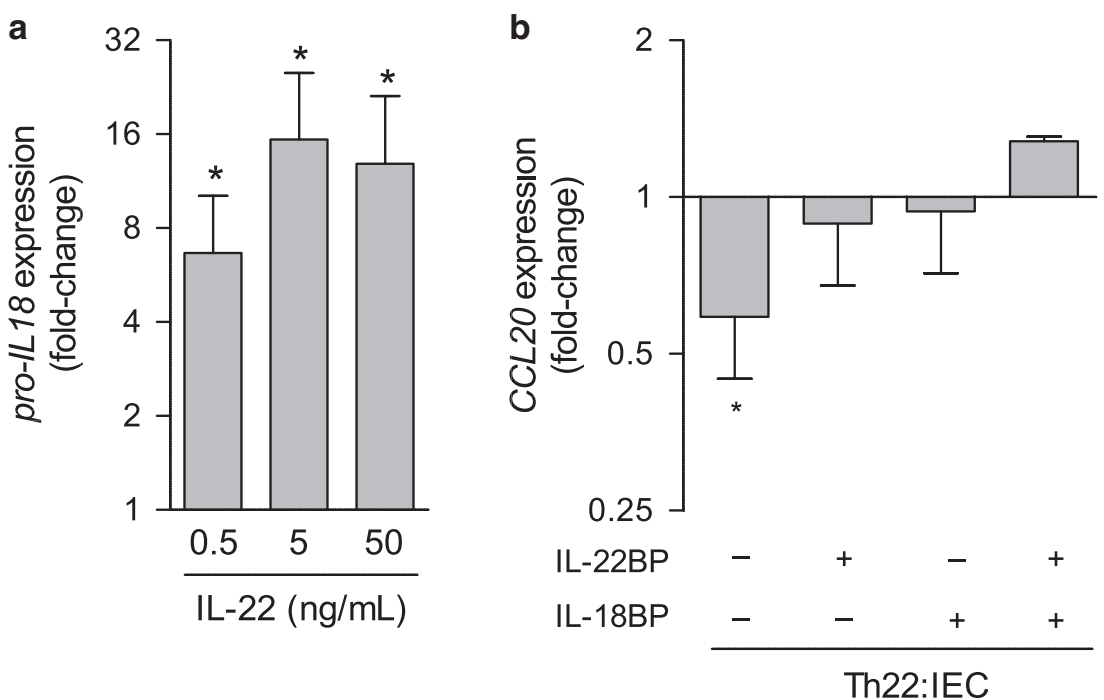

Fig. 4 Role of IL-18 in the IL-22-induced decrease of CCL20 expression by enterocytes. a Effect of IL-22 on the expression of pro-IL-18 mRNA by enterocytes. Pro-IL-18 mRNA was quantified in the enterocytes by qRT-PCR and expressed as fold change expression compared with unstimulated enterocytes (log2 scale). b Impact of IL-22BP and IL-18-BP (IL-22 and IL-18 antagonists, respectively) on CCL20 mRNA expression by enterocytes in Th22:IEC coculture. Enterocytes were cocultured with FACS-sorted Th22 or Th17 cells, added in the bottom chamber for 15 h. CCL20 mRNA was quantified in the enterocytes by qRT-PCR. CCL20 expression in Th22:IEC coculture was normalized relatively to the expression in Th17:IEC coculture (set to 1) and expressed as fold change (log2 scale). Presented data were obtained from six to ten independent experiments performed with different donors. Paired Wilcoxon's test was used to compare IL-18 expression upon stimulation vs. unstimulated condition, and to compare CCL20 expression in the various Th22:IEC coculture conditions vs. Th17:IEC coculture (intradonor pairing); $P$ value is shown above each bar; ${ }^{*} P<0.05$. IEC, intestine epithelial cells. Means and SEM are shown.

The proliferation and apoptosis rates of Th17 and Th22 cells, as indicated by expression of $\mathrm{Ki}-67$ and Annexin $\mathrm{V}$, were not significantly changed upon CCL20 and CCL28 stimulation ex vivo (Supplementary Fig. S4a, b). Thus, differences in the recruitment, rather than in proliferation and/or survival, probably best explain the impact of CCL20 and CCL28 chemokines on the observed frequencies of Th17 and Th22 cells.

Taken together, these results suggest that in a setting where CCL20 is low, such as in treated HIV-1-infected individuals, Th22 cells could use the alternative CCR10-CCL28 chemotactic axis to migrate to the gut mucosa instead of the altered CCR6-CCL20 axis. Further, Th22 cells contribute to blunted CCL20-mediated recruitment of Th17 cells in the gut mucosa by an IL-22- and IL-18dependant mechanism. This could explain why Th22 cells are better restored than Th17 cells in the gut mucosa of HIV-1infected individuals on antiretroviral therapy.

Preserved Th22 cells in the duodenal mucosa do not compensate for the loss of Th17 cells regarding microbial translocation and inflammation in treated HIV-1-infected individuals

Both Th17 and Th22 cells normally contribute to mucosal defense, by preserving the integrity of the mucosal barrier, and thus preventing microbial translocation, but their respective roles remain unclear in this setting where a preserved frequency of Th22 cells contrasts with the lack of Th17 cells.

We quantified the plasmatic concentration of sCD14 and sCD163, two systemic markers associated with microbial translocation, inflammation, and mortality in HIV-1-infected individuals. $^{33,34}$ The levels of sCD14 and sCD163 were significantly higher in treated HIV-1-infected individuals compared with uninfected controls ( $P=0.0003$ for both markers, Fig. 6a, b). The blood CD4/CD8 T-cell ratio, a parameter associated with chronic inflammation and non-AIDS-related morbidity on antiretroviral therapy, remained lower in treated HIV-1-infected individuals compared with uninfected controls $(P=0.001$, Fig. $6 \mathrm{c}) .^{35}$

The frequency of Th17 cells in the duodenal mucosa was negatively correlated with the blood concentrations of both sCD14 and sCD163 $(\rho=-0.63, P=0.003$, and $\rho=-0.75$,
$P=0.0001$, respectively; Fig. $6 \mathrm{~d}$, e), underlining the key role of gut Th17 cells in mucosal defense. The frequency of Th17 cells in the duodenal mucosa was also positively correlated with the CD4/ CD8 T-cell ratio in peripheral blood ( $\rho=0.60, P=0.007$, Fig. $6 f$ ). By contrast, the frequency of Th22 cells in the duodenal mucosa was not associated with any of these markers (Fig. $6 \mathrm{~g}-\mathrm{i}$ ). These results suggest that Th22 cells do not functionally compensate for the loss of Th17 cells regarding microbial translocation and subsequent inflammation.

\section{DISCUSSION}

Herein, we showed that Th22 cell frequency is restored in the lamina propria of the duodenal mucosa of HIV-1-infected individuals receiving prolonged effective antiretroviral therapy initiated at the chronic phase, contrasting with the persistent depletion of Th17 cells.

Th22 cells can migrate along the CCR6-CCL20 and CCR10-CCL28 chemotactic axes, while Th17 cells depend on the CCR6-CCL20 axis only. ${ }^{6,31,36}$ We previously found that CCL20 production by enterocytes is reduced in the duodenal mucosa of treated HIV-1-infected individuals, impairing the recruitment of $\mathrm{CCR6}^{+}$cells, notably Th17 cells. ${ }^{28}$ By contrast, we found here that CCL28 production by enterocytes is not altered in treated HIV-1infected individuals, and that it could allow the effective recruitment of CCR $10^{+}$cells, such as Th22 cells, in the gut as an alternative pathway to the CCR6-CCL20 axis. We found that Th22 cells preferentially used the CCR6-CCL20 axis when enough CCL20 is present in the microenvironment, but switch to the CCR10-CCL28 axis when the CCL20/CCL28 ratio drops, as it appears to be the case in the duodenal mucosa of treated HIV-1infected individuals. It could be hypothesized that there is some functional interference between CCR6 and CCR10 on Th22 cells. Heterodimerization between chemokine receptors has already been described, notably between CCR2, CCR5, and CXCR4, but CCR6/CCR10 heterodimerization remain to be demonstrated. ${ }^{37-39}$ In preliminary experiments, we observed a down-modulation of the CCR10-CCL28 chemotactic axis when CCR6 is occupied by 

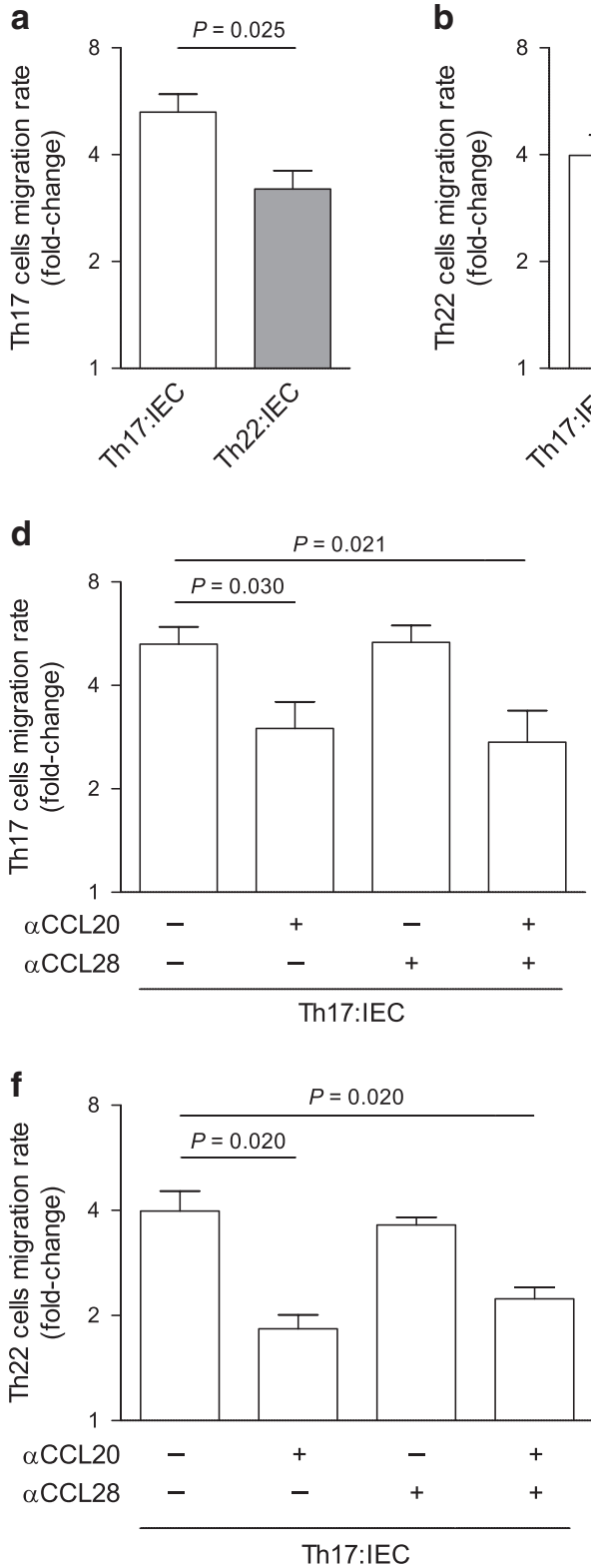

h

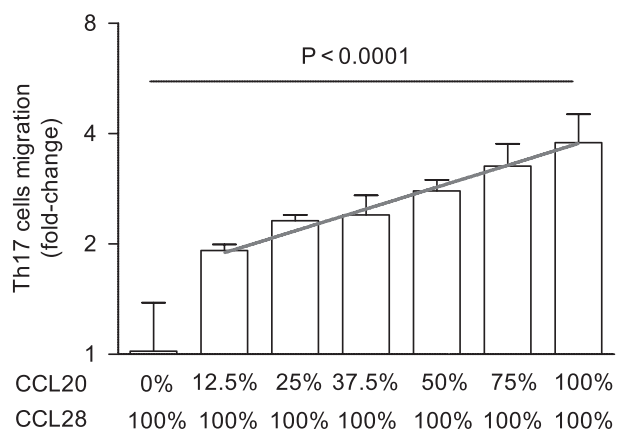

CCL20, suggesting a possible cross-desensitization, deserving further studies.

We also showed that IL-22, the main cytokine produced by Th22 cells, reduced CCL20 production in enterocytes through an indirect IL-18-mediated mechanism. The production of IL-18 by

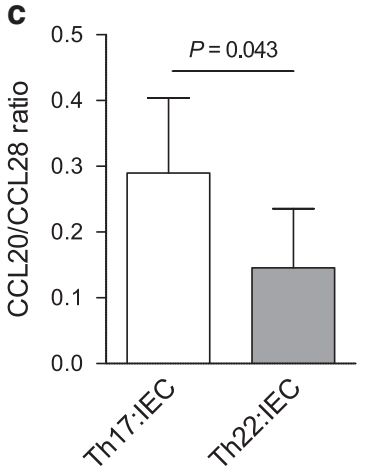

e
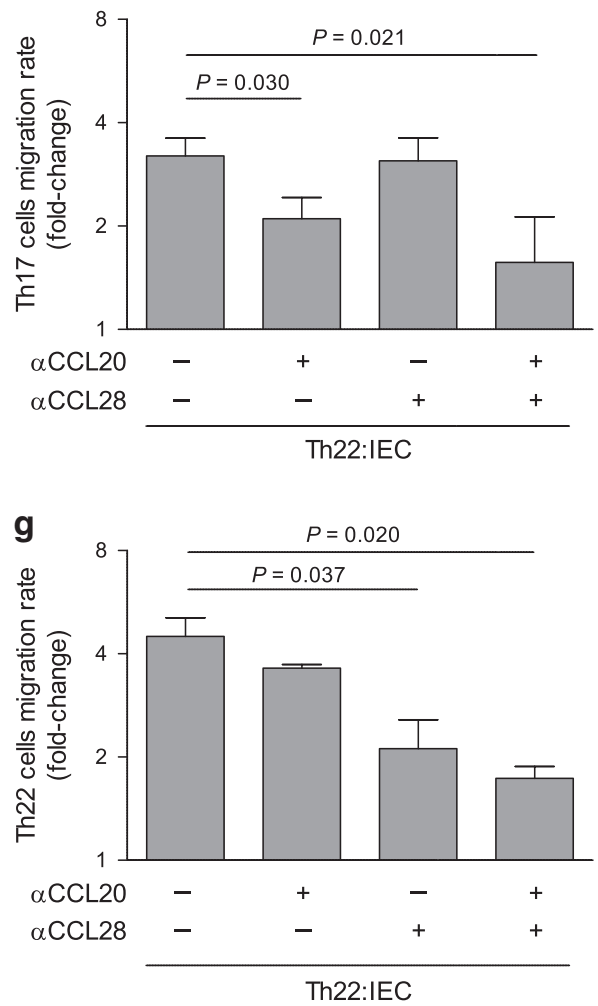

i

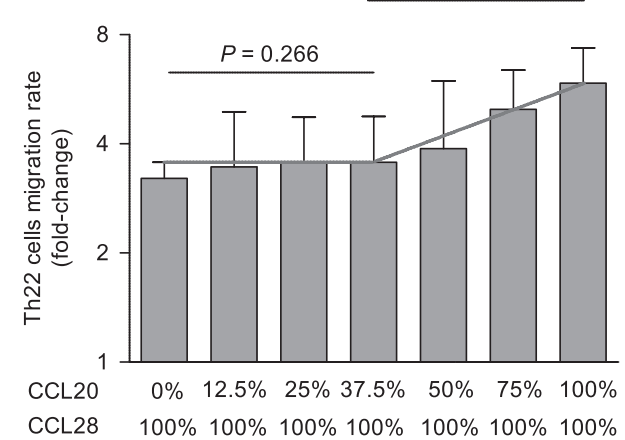

enterocytes in response to IL-22 contributes to reduced production of CCL20 by enterocytes. However, IL-18 also links Th22 to Th1 cells, as it increases the production of IFN- $\gamma$ by Th1 cells. We recently reported that Th1 cells are increased in the duodenal mucosa of treated HIV-1-infected individuals, and IFN- $\gamma$ also 
Fig. 5 Chemotaxis of Th17 and Th22 cells in response to the CCL20/CCL28 ratio in the microenvironment. Cell migration rate of a Th17 and $\mathbf{b}$ Th22 cells in response to a conditioned medium from Th17:IEC vs. Th22:IEC coculture (fold change relatively to a control medium from unstimulated enterocytes). c CCL20/CCL28 ratio in the medium of Th17:IEC vs. Th22:IEC coculture (CCL20 and CCL28 chemokines were quantified by ELISA). Cell migration rate of $\mathbf{d}$, e Th17 and $\mathbf{f}, \mathbf{g}$ Th2 22 cells in response to $\mathbf{d}, \mathbf{f}$ a conditioned medium from Th17:IEC coculture and e, $\mathbf{g}$ a conditioned medium from Th22:IEC coculture relatively to a control medium from unstimulated enterocytes. Anti-CCL20 and anti-CCL28 mAbs were used to antagonize CCL20- and CCL28-mediated chemotaxis, respectively. Cell migration rate of $\mathbf{h}$ Th17 and $\mathbf{i}$ Th22 cells in response to varying CCL20/CCL28 ratio (0:100\% to $100: 100 \%$ of maximum chemotactic activity), relatively to a control medium from unstimulated enterocytes. The number of cells migrating through the $5-\mu \mathrm{m}$-pore membrane in $15 \mathrm{~h}$ was counted. Experiments were repeated three to six times with different donors. Kruskall-Wallis's test was used for assessing a difference between all the conditions, and then paired Wilcoxon's test was used to assess differences between unblocked/blocked conditions in each experiment. IEC intestine epithelial cells. Means and SEM are shown.

contributes to reducing CCL20 production by enterocytes. ${ }^{30}$ Th22 and Th1 cells could thus synergize in blunting CCL20-mediated recruitment of Th17 cells.

Th17 and Th22 cells both contribute to maintaining the integrity of the intestinal epithelium barrier and to inducing the production of antibacterial defensins by enterocytes. ${ }^{15-17}$ However, microbial translocation events, as assessed directly by lipopolysaccharide levels, or indirectly by sCD14 and sCD163 levels in the blood, remain more frequent in treated HIV-1infected individuals than in uninfected controls. ${ }^{33,34,40}$ These findings suggest that the integrity of intestinal immune barrier is not fully restored despite prolonged antiretroviral therapy. Herein, we found that the frequencies of Th17 but not Th22 cells in the duodenal mucosa were negatively correlated with sCD14 and SCD163 levels in peripheral blood, and positively correlated with the CD4/CD8 T-cell ratio. These results suggest that the reconstitution of Th22 cells does not functionally compensate for the persistent depletion of Th17 cells in the gut mucosa.

Whether residual HIV-1 infection events could also contribute to the preferential depletion of Th17 cells in treated HIV-1-infected individuals remains to be seen. In preliminary experiments of HIV1 infection of gut histocultures ex vivo, Th22 cells seem less susceptible to infection by R5-tropic viruses than Th17 cells, with HIV-1 DNA levels being about 0.5 log lower in Th22 compared with Th17 cells (data not shown). Th22 cells in the duodenal mucosa express slightly lower levels of CCR5, the main coreceptor for HIV1 entry, compared with Th17 cells (data not shown). The transcription factor AhR could play a role in reducing the susceptibility of Th22 cells to HIV-1 infection, but this has only been demonstrated in macrophages to date. ${ }^{41}$ The mechanisms involved in, and the consequences of, the possible reduced susceptibility of Th22 cells to HIV-1 infection demand further studies. Difference in cell-associated HIV-1 DNA levels between gut Th17 and Th22 cells in HIV-1-infected individuals should be investigated.

Most studies on mucosal Th17 cells in HIV-1-infected individuals have been performed on samples from the rectum or the distal colon, and the cervix, while we studied here the duodenal mucosa. ${ }^{10,13,25,42,43}$ The various segments of the gastrointestinal tract present significant differences regarding the composition of the gut microbiota and metabolites and their interactions with the epithelium, depending notably on the presence or not of a mucus layer, as in the colon but not in the small intestine. But there are also differences regarding the chemotactic axes involved in immune cells recruitment, and the frequencies of the different T-cell subsets, with maximum frequency of Th17 cells in the small intestine. ${ }^{44}$ The conclusions drawn from studies sampled at different levels of the gastrointestinal tract could thus differ significantly.

In conclusion, we demonstrated here that Th22 cells could use the CCR10-CCL28 chemotactic axis to migrate in the duodenal mucosa, as an alternative to the defective CCR6-CCL20 axis. Th22 cells also contribute to reduced CCL20 production in enterocytes through an IL-22- and IL-18-dependent mechanism. This could contribute to the better reconstitution of Th22 than Th17 cells on antiretroviral therapy. However, residual microbial translocation and inflammation persist in treated HIV-1-infected individuals.

\section{METHODS}

Subjects and samples

The ANRS EP61 GALT study recruited HIV-1-infected individuals on prolonged effective antiretroviral therapy and uninfected controls at Toulouse University Hospital, France. Herein, we studied ten HIV-1-infected individuals and ten uninfected controls for whom biopsies of duodenal mucosa and blood samples had been collected. The HIV-1-infected individuals received combined antiretroviral therapy initiated at the chronic stage of infection, with a median nadir $\mathrm{CD}^{+}$T-cells count of 240 cells $/ \mu \mathrm{l}$ (interquartile range $[\mathrm{IQR}], 188-250$ cells $/ \mu \mathrm{L})$. They maintained sustained undetectable plasma HIV-1 RNA load ( $<30$ copies $/ \mathrm{mL}$ ) on continuous antiretroviral therapy for a median of 4.3 years (IQR, 3.1-8.9 years). Their median $\mathrm{CD}^{+}{ }^{+} \mathrm{T}$-cells count at sampling time was of 734 cells $/ \mu \mathrm{L}$ (IQR, 612-903 cells $/ \mu \mathrm{L})$. All individuals were free of inflammatory or lymphoproliferative bowel disease on histopathologic examination. Clinical characteristics for HIV-1infected individuals and uninfected controls are summarized in Supplementary Tables S1 and S2, respectively.

Ethics statement

The study was approved by the Institutional Review Board CPP Sud-Ouest et Outre-Mer I. All participants provided informed consent (trial registration number NCT02906137).

Isolation of $\mathrm{CD}^{+} \mathrm{T}$ lymphocytes from the intestine mucosa Duodenal biopsies ( $n=5$, pooled) were digested with $0.5 \mathrm{mg} / \mathrm{mL}$ collagenase type II-S (Sigma-Aldrich). T lymphocytes were isolated by positive selection (EasySep Human CD2 Positive Selection Kit, Stemcell).

Immunophenotyping of T lymphocytes

Flow cytometry analyses were performed on a BD Fortessa driven by the FACSDiva software (BD Biosciences). Th17 (CD3 ${ }^{+} \mathrm{CD} 4^{+}$ $\left.\mathrm{CXCR}^{-} \mathrm{CCR}^{+}{ }^{+} \mathrm{CCR}^{+} \mathrm{CD} 161^{+}\right), \mathrm{Th} 22\left(\mathrm{CD}^{+} \mathrm{CD}^{+}{ }^{+} \mathrm{CXCR}^{-}{ }^{-} \mathrm{CCR} 4^{+}\right.$ $\left.\mathrm{CCR}^{+} \mathrm{CD} 161^{-} \mathrm{CCR}_{10}{ }^{+}\right)$, Th1 $\left(\mathrm{CD}^{+}{ }^{+} \mathrm{CD} 4^{+} \mathrm{CXCR}^{+}{ }^{+} \mathrm{CCR} 4^{-} \mathrm{CCR}^{-}\right)^{-}$, Th1Th17 $\left(\mathrm{CD}^{+}{ }^{+} \mathrm{CD} 4^{+} \mathrm{CXCR} 3^{+} \mathrm{CCR} 4^{-} \mathrm{CCR} 6^{+} \mathrm{CD} 161^{+}\right)$, and Th2 $\left(\mathrm{CD}^{+}{ }^{+} \mathrm{CD} 4^{+} \mathrm{CXCR}^{-}{ }^{-} \mathrm{CCR} 4^{+} \mathrm{CCR} 6^{-}\right)$were stained with anti-human CD3-BV650 (clone OKT3) (Biolegend); anti-CD4-PECy7 (SK3), anti-CCR4-PE (1G1), anti-CCR6-BV786 (11A9) (all from BD Biosciences); CD161-eFluor450 (HP-3G10) (eBiosciences); anti-CXCR3PE-CF594 (REA232), and anti-CCR10-APC (REA326) (Miltenyi) mAbs. Gating strategies are shown in Fig. 1 for Th17 and Th22 cells, and in Supplementary Fig. S1 for Th1, Th1Th17, and Th2 cells.

Immunohistochemistry

Fresh tissues were fixed in $4 \%$ neutral buffered formalin and embedded in paraffin. Sections $(3 \mu \mathrm{m})$ were stained with hematoxylin and eosin. Immunohistochemistry for CCL28 was performed using a mouse anti-human CCL28 mAb (62714, R\&D) and a goat anti-mouse $\lg \mathrm{G}(\mathrm{H}+\mathrm{L})$ highly cross-adsorbed secondary antibody 

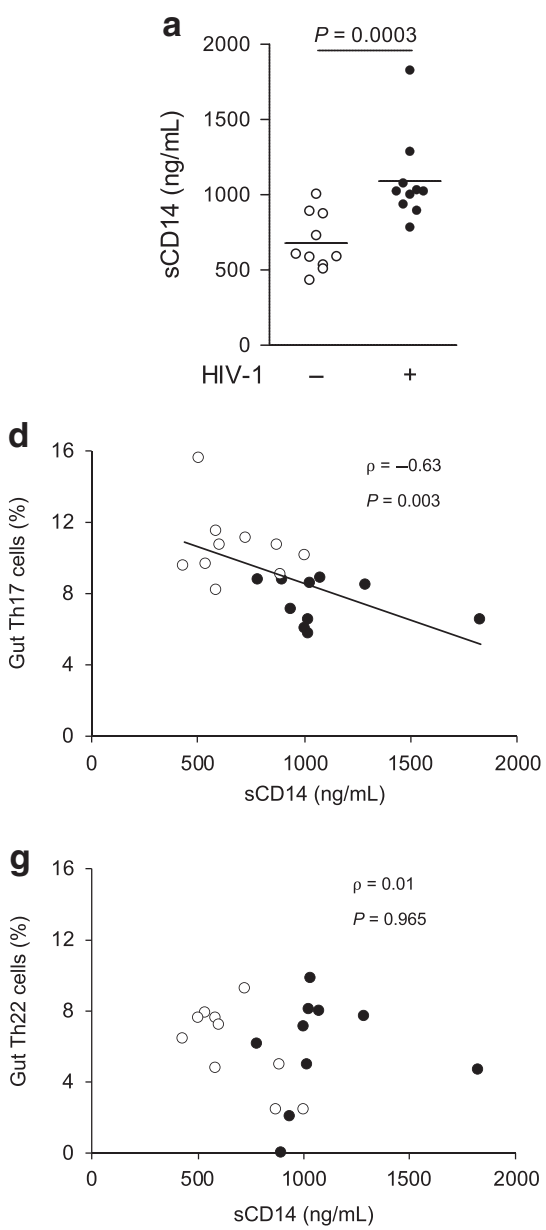
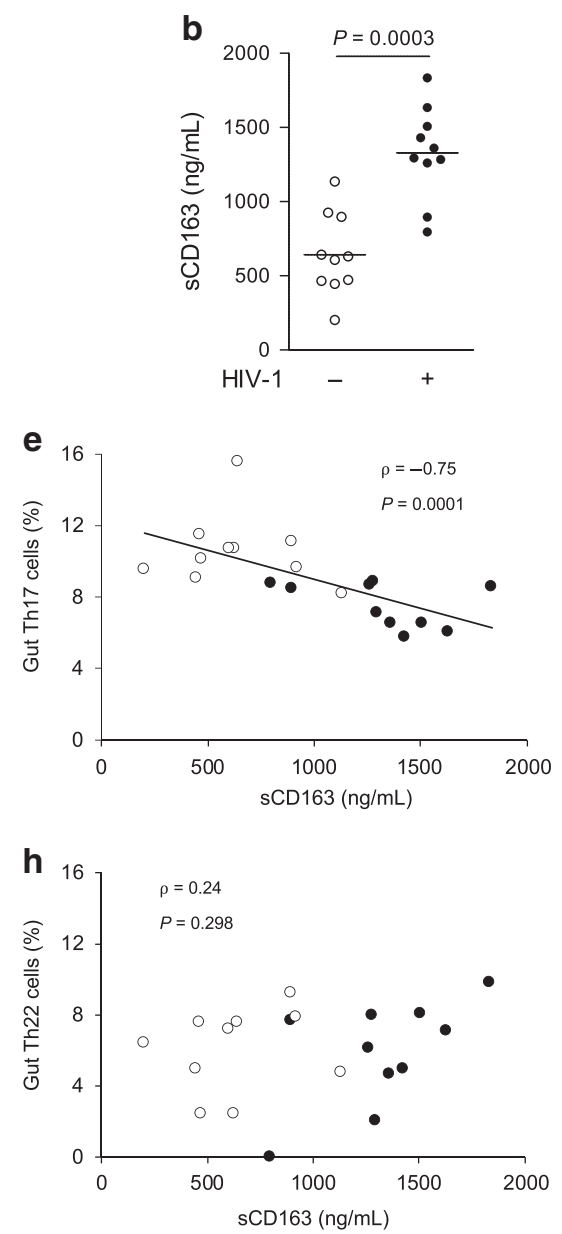
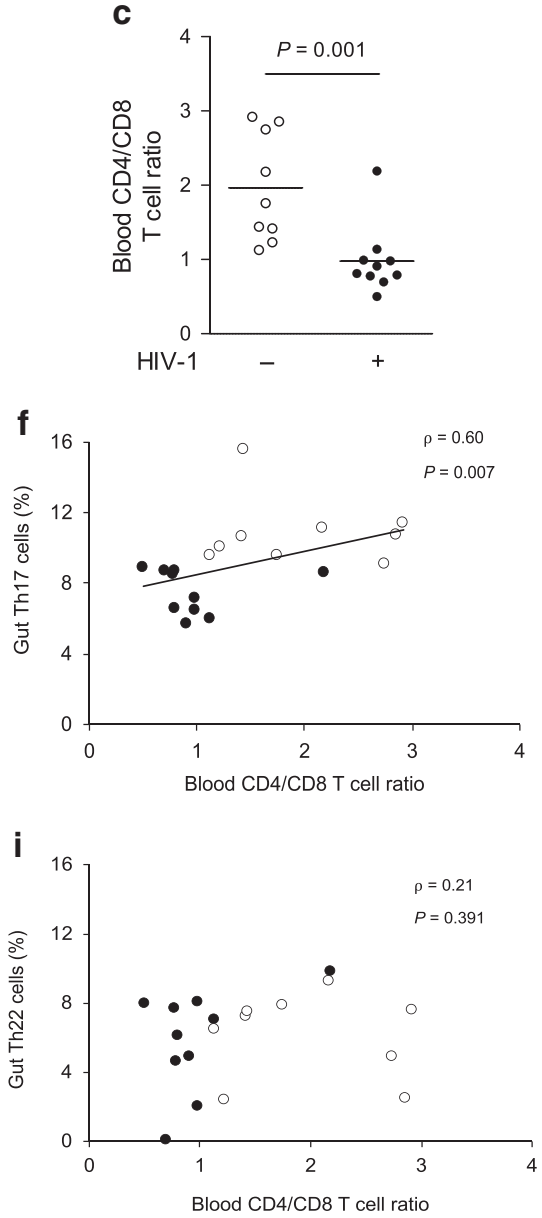

Fig. 6 Correlations between the frequencies of Th17 and Th22 cells in the duodenal mucosa and systemic inflammatory markers. Levels of a sCD14, b sCD163, and c CD4/CD8 T-cells ratio in peripheral blood of treated HIV-1-infected $(n=10)$ and uninfected individuals $(n=10$ for sCD14 and sCD163; $n=9$ for the CD4/CD8 T-cell ratio). SCD14 and SCD163 were quantified in plasma by ELISA. The CD4/CD8 T-cells ratio was measured in peripheral blood by flow cytometry. Groups were compared with the Wilcoxon's rank-sum test. Median bars are shown. Correlations between the frequency of Th17 cells in the duodenal mucosa and $\mathbf{d}$ sCD14, e sCD163, and $\mathbf{f}$ CD4/CD8 T-cells ratio in peripheral blood. Lack of correlation between the frequency of Th22 cells in the duodenal mucosa and $\mathbf{g}$ sCD14, $\mathbf{h}$ sCD163, and i CD4/CD8 T-cells ratio in peripheral blood. ( $n=10$ treated HIV-1-infected and $n=10$ uninfected individuals for SCD14 and sCD163 or $n=9$ uninfected individuals for the CD4/CD8 T-cell ratio). Each symbol represents one individual. HIV $-1^{-}$, white symbol; $\mathrm{HIV}-1^{+}$, black symbol. Correlations were estimated by calculating Spearman's rank correlation coefficients.

coupled with Alexa Fluor Plus 555 (Thermo Fisher) on an ApoTome (Zeiss, original magnification $\times 63$ ). CCL28 production by the epithelium was quantified using NIS-element (Nikon) and the Zen software in a blinded fashion regarding HIV-1 infection status.

Quantification of sCD14 and sCD163

Soluble CD14 (sCD14) and sCD163 were quantified in plasma by ELISA (DC140, R\&D systems; IQP-383, Eurobio, respectively).

Isolation, culture, and differentiation of IEC

IEC were isolated from upper small bowel surgical resections obtained from individuals free of HIV infection and inflammatory bowel diseases. Intestinal tissue was digested using collagenase, dispase, and DNAse. Epithelial cells were subsequently isolated by magnetic positive selection (Human EpCAM positive selection kit, Stemcell). Cell purity was $>95 \%$ by flow cytometry (anti-EpCAM $\mathrm{mAb}$, Stemcell). Cells were placed in gelatin-coated wells (Corning BioCoat) and cultured in DMEM:F-12 (1:1), 5\% FBS, 1\% PSA, supplemented with HCM Single Quote kit (Lonza). Epithelial cells were cultured and expanded ex vivo up to five passages. For experimental purposes, primary IEC were cultured and differentiated on transwell inserts $(0.4-\mu \mathrm{m}$-pore polycarbonate membranes coated with Corning Matrigel) at $4 \times 10^{5}$ cells per insert in DMEM:F-12 (1:1), $5 \%$ FBS, 1\% PSA, with ITS media supplement (Sigma-Aldrich) and 5 $\mathrm{ng} / \mathrm{mL}$ EGF for 2 days. FBS was then removed from the medium, while hydrocortisone and 3,3',5-triiodo-L-thyronine (Sigma-Aldrich) were added. This resulted in monolayers of differentiated epithelial cells expressing cytokeratin (CK)-18 and CK-20, EpCAM, and tight junctions revealed by continuous ZO-1 staining, as already published. $^{28}$ The integrity of the epithelium was checked by measuring the transepithelial electrical resistance between the apical and basolateral sides of the monolayer (mean: $450 \Omega / \mathrm{cm}^{2}$, as assessed using Millicell ERS-2, Millipore).

Ex vivo stimulation of primary IEC by cytokines

Primary IEC cultured on transwell inserts were stimulated for $15 \mathrm{~h}$ with 0.5 , 5 , or $50 \mathrm{ng} / \mathrm{mL}$ of human IL-17A, IL-17F, IL-22, TNF-a, and IL-1 $\beta$ (Miltenyi) added into the bottom chamber.

Th17 and Th22 cell sorting and coculture with primary IEC Th17 and Th22 cells were sorted by flow cytometry on a FACSARIA II (BD Biosciences) from PBMCs of uninfected donors. Gating strategy is shown in Fig. 1. Purity of sorted Th17 and Th22 cells was $>99 \%$ (data not shown). Sorted Th17 or Th22 cells were 
expanded ex vivo for 5-7 days following CD3/CD28 stimulation (Dynabeads, Thermo Fisher) in CTS OpTmizer medium (Thermo Fisher). To maintain their polarization, Th17 cells were expanded in medium supplemented with IL-2 $(50 \mathrm{UI} / \mathrm{mL}), \mathrm{IL}-1 ß(10 \mathrm{ng} / \mathrm{mL})$, and IL-23 (10 ng/mL; all from Miltenyi). Th22 cells were expanded in medium supplemented with $\mathrm{IL}-2(50 \mathrm{UI} / \mathrm{mL}), \mathrm{IL}-6(20 \mathrm{ng} / \mathrm{mL})$, and TNF-a ( $50 \mathrm{ng} / \mathrm{mL}$; all from Miltenyi). The Th17 and Th22 phenotype was maintained regarding chemokine receptors expression, as assessed by flow cytometry, when compared with freshly sorted cells (data not shown). IL-17A and IL-22 production was quantified by ELISA (R\&D Systems). AhR and RORc transcription factors expression was quantified in sorted cells by qRT-PCR (Taqman gene expression assays, Thermo Fisher). Th17 and Th22 cells were activated with PMA/ionomycin for $6 \mathrm{~h}$ before being added to the bottom chamber of transwells for coculture with IEC $\left(1 \times 10^{5}\right.$ Th cells: $\left.4 \times 10^{5} \mathrm{IEC}\right)$ during $15 \mathrm{~h}$. IL-18BP $(1 \mu \mathrm{g} / \mathrm{mL}$, R\&D systems) and IL-22BP $(0.2 \mu \mathrm{g} / \mathrm{mL}$, R\&D systems) were used to antagonize the biological effect of IL-18 and/or IL-22, respectively.

Measure of the proliferation and apoptosis rates of Th17 and Th22 cells

Th17 and Th22 cells were cultured overnight in presence of CCL20 and/or CCL28 recombinant proteins (R\&D Systems) and stained with Annexin V-FITC and Propidium lodide (556547, BD Biosciences) to measure apoptosis, and with anti-Ki67-FITC (556026, $\mathrm{BD}$ Biosciences) to measure proliferation, by flow cytometry.

RT-PCR quantification of chemokine mRNAs

RNA was extracted from primary IEC using the RNeasy minikit (Qiagen). CCL20, CCL28, and prolL-18 mRNA quantification was performed on a LightCycler 480 (Roche) ( $\Delta \Delta C$ t method) using primers designed to amplify a fragment encompassing a spliced region of mRNA. Expression was normalized to GAPDH mRNA.

Quantification of chemokines

CCL20 and CCL28 were quantified by ELISA (R\&D Systems) in the medium of coculture experiments.

Cell migration assay

Sorted Th17 and Th22 cells were loaded in the upper chamber of a 24-well transwell plate ( $5 \mu \mathrm{m}$ polycarbonate membranes) at $3 \times$ $10^{5}$ Th cells in $150 \mu \mathrm{L}$ of CTS OpTmizer medium (Life Technology). Control medium from unstimulated enterocytes or conditioned medium from Th17:IEC or Th22:IEC cocultures was loaded in the bottom chamber. Anti-CCL20 (10 $\mu \mathrm{g} / \mathrm{mL}, \mathrm{MAB} 360, \mathrm{R} \& D$ Systems) or anti-CCCL28 $(10 \mu \mathrm{g} / \mathrm{mL}, \mathrm{MAB717}, \mathrm{R} \& D$ Systems $) \mathrm{mAbs}$ were used to antagonize CCL20- and CCL28-driven chemotaxis, respectively. In gradient experiments, CCL20 and CCL28 recombinant proteins (R\&D Systems) were loaded in the bottom chamber with a CCL20/CCL28 ratio varying from $0: 100 \%$ to $100: 100 \%$ of their maximum chemotactic activity $(10$ and $200 \mathrm{ng} / \mathrm{mL}$, respectively). Migration assay was performed overnight at $37^{\circ} \mathrm{C}$, and then cells in the lower chamber were counted.

\section{Statistical analyses}

Quantitative variables were compared using the Kruskall-Wallis's or the Wilcoxon's rank-sum test. Correlations were estimated by calculating Spearman's rank correlation coefficients. Cuzick's test was used to assess for a trend across ordered groups. Paired Wilcoxon's test was used for intradonor comparisons between different conditions in IEC cultures. All tests were two-sided, and $P$ values $<0.05$ were considered statistically significant. Statistical analyses were performed with Stata 16.0.

\section{ACKNOWLEDGEMENTS}

This project was supported by grants from the French National Agency for Research on AIDS and Viral Hepatitis, Sidaction, and the Institut Universitaire de France. We are indebted to the patients who took part in this study. We also thank S. Lagarrigue, A. Frelat, and C. Pomes for their help in monitoring the study; A-L. Iscache, L. De La Fuente, V. Duplan-Eche, and F.-E. L'Faqihi for their help in flow cytometry cell sorting; F. Capilla and A. Alloy for her help in immunohistochemistry; and S. Allart, D. Daviaud, and A. Canivet for their help in microscopy; and Martha M. Cooper for checking the English text.

\section{AUTHOR CONTRIBUTIONS}

M.N. contributed to the project design, set up methods, and performed the experiments; P.D., G.M-B., B.S., N.C., K.B., and L.A. recruited participants and provided samples; M.N., M.R., C.L., and M.C., processed the samples and performed the experiments; M.N., M.R., J.I., and P.D. analyzed and interpreted the data; M.N. and P.D. wrote the manuscript; C.L., G.M.-B., and J.I. reviewed the draft; P.D. conceived the project and directed the research.

\section{FUNDING}

Agence Nationale de Recherches sur le Sida et les Hépatites Virales (ANRS EP61 GALT study), Sidaction, and the Institut Universitaire de France. This work was partly presented at the 26th Conference on Retroviruses and Opportunistic Infections, Seattle, WA, USA, March 4-7, 2019, Abstract 203.

\section{ADDITIONAL INFORMATION}

The online version of this article (https://doi.org/10.1038/s41385-020-0286-6) contains supplementary material, which is available to authorized users.

Competing interests: The authors declare no competing interests.

Publisher's note Springer Nature remains neutral with regard to jurisdictional claims in published maps and institutional affiliations.

\section{REFERENCES}

1. Brenchley, J. M. et al. CD4+ T cell depletion during all stages of HIV disease occurs predominantly in the gastrointestinal tract. J. Exp. Med. 200, 749-759 (2004).

2. Mehandru, S. et al. Primary HIV-1 infection is associated with preferential depletion of $\mathrm{CD} 4+\mathrm{T}$ lymphocytes from effector sites in the gastrointestinal tract. J. Exp. Med. 200, 761-770 (2004).

3. Guadalupe, M. et al. Severe CD4+ T-cell depletion in gut lymphoid tissue during primary human immunodeficiency virus type 1 infection and substantial delay in restoration following highly active antiretroviral therapy. J. Virol. 77, 11708-11717 (2003).

4. Brenchley, J. M. et al. Microbial translocation is a cause of systemic immune activation in chronic HIV infection. Nat. Med. 12, 1365-1371 (2006).

5. Mudd, J. C. \& Brenchley, J. M. Gut mucosal barrier dysfunction, microbial dysbiosis, and their role in HIV-1 disease progression. J. Infect. Dis. 214(Suppl 2), S58-S66 (2016).

6. Annunziato, F. et al. Phenotypic and functional features of human Th17 cells. J. Exp. Med. 204, 1849-1861 (2007).

7. Brenchley, J. M. et al. Differential Th17 CD4 T-cell depletion in pathogenic and nonpathogenic lentiviral infections. Blood 112, 2826-2835 (2008).

8. El Hed, A. et al. Susceptibility of human Th17 cells to human immunodeficiency virus and their perturbation during infection. J. Infect. Dis. 201, 843-854 (2010).

9. Gosselin, A. et al. Peripheral blood CCR4+CCR6+ and CXCR3+CCR6+CD4+ $\mathrm{T}$ cells are highly permissive to HIV-1 infection. J. Immunol. 184, 1604-1616 (2010).

10. Gosselin, A. et al. HIV persists in CCR6+CD4+ T cells from colon and blood during antiretroviral therapy. AIDS 31, 35-48 (2017).

11. Wacleche, V. S. et al. New insights into the heterogeneity of Th17 subsets contributing to HIV-1 persistence during antiretroviral therapy. Retrovirology 13, 59 (2016).

12. Raffatellu, M. et al. Simian immunodeficiency virus-induced mucosal interleukin17 deficiency promotes Salmonella dissemination from the gut. Nat. Med. 14, 421-428 (2008).

13. Chege, D. et al. Sigmoid Th17 populations, the HIV latent reservoir, and microbial translocation in men on long-term antiretroviral therapy. AIDS 25, 741-749 (2011).

14. Kim, C. J. et al. Mucosal Th17 cell function is altered during HIV infection and is an independent predictor of systemic immune activation. J. Immunol. 191, 2164-2173 (2013). 
15. Eyerich, S. et al. Th22 cells represent a distinct human T cell subset involved in epidermal immunity and remodeling. J. Clin. Investig. 119, 3573-3585 (2009).

16. Fernandes, S. M. et al. Enteric mucosa integrity in the presence of a preserved innate interleukin 22 compartment in HIV type 1-treated individuals. J. Infect. Dis. 210, 630-640 (2014).

17. Kim, C. J. et al. A role for mucosal IL-22 production and Th22 cells in HIVassociated mucosal immunopathogenesis. Mucosal Immunol. 5, 670-680 (2012).

18. Ivanov, I. I. et al. Induction of intestinal Th17 cells by segmented filamentous bacteria. Cell 139, 485-498 (2009).

19. Sano, T. et al. An IL-23R/LL-22 circuit regulates epithelial serum amyloid $A$ to promote local effector Th17 responses. Cell 163, 381-393 (2015).

20. Lee, J. Y. et al. Serum amyloid A proteins induce pathogenic Th17 cells and promote inflammatory disease. Cell 180, 79-91.e16 (2020).

21. Acosta-Rodriguez, E. V. et al. Surface phenotype and antigenic specificity of human interleukin 17-producing $\mathrm{T}$ helper memory cells. Nat. Immunol. 8, 639-646 (2007).

22. Cosmi, L. et al. Human interleukin 17-producing cells originate from a CD161 +CD4+ T cell precursor. J. Exp. Med 205, 1903-1916 (2008).

23. Trifari, S., Kaplan, C. D., Tran, E. H., Crellin, N. K. \& Spits, H. Identification of a human helper $\mathrm{T}$ cell population that has abundant production of interleukin 22 and is distinct from $\mathrm{T}(\mathrm{H})-17, \mathrm{~T}(\mathrm{H}) 1$ and $\mathrm{T}(\mathrm{H}) 2$ cells. Nat. Immunol. 10, 864-871 (2009).

24. Page, E. E. et al. Loss of Th22 cells is associated with increased immune activation and IDO-1 activity in HIV-1 infection. J. Acquir Immune Defic. Syndr. 67, 227-235 (2014).

25. Ryan, E. S. et al. Loss of function of intestinal IL-17 and IL-22 producing cells contributes to inflammation and viral persistence in SIV-infected rhesus macaques. PLoS Pathog. 12, e1005412 (2016).

26. Kok, A. et al. Early initiation of combined antiretroviral therapy preserves immune function in the gut of HIV-infected patients. Mucosal Immunol. 8, 127-140 (2015).

27. Schuetz, A. et al. Initiation of ART during early acute HIV infection preserves mucosal Th17 function and reverses HIV-related immune activation. PLoS Pathog. 10, e1004543 (2014)

28. Loiseau, C. et al. CCR6(-) regulatory T cells blunt the restoration of gut Th17 cells along the CCR6-CCL20 axis in treated HIV-1-infected individuals. Mucosal Immunol. 9, 1137-1150 (2016).

29. Micci, L. et al. Interleukin-21 combined with ART reduces inflammation and viral reservoir in SIV-infected macaques. J. Clin. Investig. 125, 4497-4513 (2015).
30. Loiseau C. et al. Increase of CXCR3 + T cells impairs Th17 cells recruitment in the small intestine mucosa through IFN-gamma and IL-18 during treated HIV-1 infection. J. Infect. Dis. 220, 830-840 (2019).

31. Pan, J. et al. A novel chemokine ligand for CCR10 and CCR3 expressed by epithelial cells in mucosal tissues. J. Immunol. 165, 2943-2949 (2000).

32. Munoz, M. et al. Interleukin-22 induces interleukin-18 expression from epithelial cells during intestinal infection. Immunity 42, 321-331 (2015).

33. Sandler, N. G. et al. Plasma levels of soluble CD14 independently predict mortality in HIV infection. J. Infect. Dis. 203, 780-790 (2011).

34. Knudsen, T. B. et al. Plasma soluble CD163 level independently predicts all-cause mortality in HIV-1-infected individuals. J. Infect. Dis. 214, 1198-1204 (2016).

35. Serrano-Villar, S. et al. HIV-infected individuals with low CD4/CD8 ratio despite effective antiretroviral therapy exhibit altered T cell subsets, heightened CD8+ T cell activation, and increased risk of non-AIDS morbidity and mortality. PLOS Pathog. 10, e1004078 (2014).

36. Kunkel, E. J. et al. CCR10 expression is a common feature of circulating and mucosal epithelial tissue IgA Ab-secreting cells. J. Clin. Investig. 111, 1001-1010 (2003).

37. Mellado, M. et al. Chemokine receptor homo- or heterodimerization activates distinct signaling pathways. EMBO J. 20, 2497-2507 (2001).

38. Sohy, D., Parmentier, M. \& Springael, J. Y. Allosteric transinhibition by specific antagonists in CCR2/CXCR4 heterodimers. J. Biol. Chem. 282, 30062-30069 (2007).

39. Isik, N., Hereld, D. \& Jin, T. Fluorescence resonance energy transfer imaging reveals that chemokine-binding modulates heterodimers of CXCR4 and CCR5 receptors. PLOS ONE 3, e3424 (2008).

40. Mavigner, $M$. et al. Altered CD4 $+\mathrm{T}$ cell homing to the gut impairs mucosal immune reconstitution in treated HIV-infected individuals. J. Clin. Investig. 122, 62-69 (2012).

41. Kueck, T., Cassella, E., Holler, J., Kim, B. \& Bieniasz, P. D. The aryl hydrocarbon receptor and interferon gamma generate antiviral states via transcriptional repression. elife 7, e38867 (2018).

42. McKinnon, L. R. et al. Characterization of a human cervical CD4+ T cell subset coexpressing multiple markers of HIV susceptibility. J. Immunol. 187, 6032-6042 (2011).

43. Joag, V. R. et al. Identification of preferential CD4+ T-cell targets for HIV infection in the cervix. Mucosal Immunol. 9, 1-12 (2016).

44. Mowat, A. M. \& Agace, W. W. Regional specialization within the intestinal immune system. Nat. Rev. Immunol. 14, 667-685 (2014). 